\title{
Equações diferenciais aplicadas ao pêndulo com massa dependente do tempo: estudo de massa com variação exponencial e polinomial
}

\section{Differential equations applied to the pendulum with time-dependent mass: study of mass with exponential and polynomial variation}

\author{
Otávio Paulino Lavor \\ Universidade Federal Rural do Semi-árido (UFERSA), \\ Departamento de Ciências Exatas e Naturais (DECEN), Pau dos Ferros, RN, Brasil \\ http://orcid.org/0000-0001-5237-3392, otavio.lavor@ufersa.edu.br
}

Antônio Nunes de Oliveira Instituto Federal de Educação, Ciência e Tecnologia do Ceará (IFCE), Campus Cedro, Cedro, CE, Brasil

http://orcid.org/0000-0001-5697-8110, nunes.vieira@ifce.edu.br

\section{Informações do Artigo}

Como citar este artigo

LAVOR, Otávio Paulino;

OLIVEIRA, Antônio Nunes de. Equações diferenciais aplicadas ao pêndulo com massa dependente do tempo: estudo de massa com variação exponencial e polinomial. REMAT: Revista Eletrônica da Matemática, Bento Gonçalves, RS, v. 7, n. 1, p. e3001, 05 jan. 2021. DOI:

https://doi.org/10.35819/remat2021 v7i1id4164.

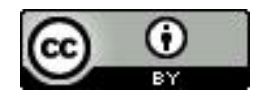

Histórico do Artigo

Submissão: 28 de abril de 2020.

Aceite: 22 de junho de 2020 .

Palavras-chave

Amortecimento

Equação de Bessel

Substituição de Variáveis

\section{Resumo}

As equações diferenciais são um dos conteúdos aplicados em diversas áreas. Na Física, uma das aplicações é o pêndulo simples, que tem oscilação independente da massa, quando esta é constante. No entanto, quando a massa não é constante, a variação de momento linear deve ser reescrita. Neste trabalho, propõe-se dois tipos de massa variável, como função exponencial e em termos de potências da variável tempo. Nos casos de ganho de massa na variação exponencial, há amortecimento que é mostrado pelos gráficos de suas soluções. Quando a massa é escrita em termos de potências, após substituição de variáveis, o problema fica modelado pela equação de Bessel, que tem ordem dependente da potência empregada na função massa. Ao final, foi verificada a participação da massa no amortecimento e os problemas analisados se mostram como aplicações que enriquecem o campo de estudo das equações diferenciais.
Keywords

Damping

Bessel Equation

Substitution of Variables

\begin{abstract}
Differential equations are one of the contents that are applied in several areas. In Physics, one of the applications is the simple pendulum that has oscillation independent of the mass, when it is constant. However, when the mass is no-constant, the variation of linear momentum must be rewritten. In this work, two types of variable mass are proposed, as an exponential function and in terms of the time variable powers. In cases of gain of mass in the exponential variation, there is damping that is shown by the graphs of their solutions. When the mass is written in terms of powers, after substitution of variables, the problem is modeled by the Bessel Equation which has a dependent order of the power used in the mass function. At the end, the participation of the mass in the damping was verified and the analyzed problems are shown as applications that enrich the differential equations study field.
\end{abstract}




\section{Introdução}

A Matemática, assim como a Física, lida muitas vezes com objetos de grande interesse prático, cuja compreensão permitiu ao homem chegar no patamar tecnológico e social de hoje. A solução de um problema físico passa inicialmente pela observação do fenômeno, seguida da construção de hipóteses/experimentação e, inevitavelmente, pelo processo de modelamento matemático, em que as equações contribuem significativamente para a compreensão do problema.

Modelos matemáticos para problemas físicos vêm sendo desenvolvidos há séculos e existe uma grande quantidade deles, em que são discutidos os casos de não homogeneidade, seja em mecânica, eletricidade ou outra área da Física. Nesse sentido, o estudo do pêndulo com massa variável dependente do tempo é um assunto que relaciona as equações diferenciais aos modelos físicos e matemáticos para objetos que executam movimentos oscilatórios, os chamados osciladores harmônicos.

Os osciladores harmônicos são objetos físicos descritos por modelos matemáticos que envolvem equações diferenciais e podem ser simples, amortecidos ou forçados. O primeiro caso tem muitas aplicações práticas e possui a descrição matemática mais simples. Segundo Thornton e Marion (2014), o Oscilador Harmônico Simples (OHS) tem equação de movimento obtida a partir da lei de Hooke. Esta equação é diferencial linear e homogênea de segunda ordem com coeficientes constantes, em que a derivada de primeira ordem não está presente. O movimento descrito por um Oscilador Harmônico Simples é denominado de Movimento Harmônico Simples (MHS).

O amortecimento ocorre devido à atuação de forças dissipativas, como afirmado por Silva e Helayël Neto (2016). Casos em que a força dissipativa é proporcional à velocidade são comuns em situações que envolvem corpos movendo-se em fluidos e um exemplo típico é o de uma gota de água deslocando-se no ar. O caso forçado envolve situações em que uma força externa atua fornecendo energia ao oscilador.

Fenômenos oscilatórios são encontrados em todas as áreas da Física, além disso, existe uma diversidade de outros sistemas cujo comportamento matemático é oscilatório. Na Biologia, o estudo das oscilações é empregado para descrever a interação entre bactérias e um vírus bacteriófago (LENSKI, 1988); na Biofísica, usa-se um modelo oscilatório para se descrever a potência mecânica envolvida na locomoção humana (ROMEO, 2009); na Economia, modelos oscilatórios são usados como inspiração para reinterpretar a teoria do crescimento econômico (SALAMANGA, 2013); na Cosmologia, são usados para descrever o comportamento ao longo do tempo, do ínflaton, um campo escalar que modula a aceleração e a desaceleração do universo (LINDE, 2000). Destaca-se ainda uma importante aplicação dos osciladores harmônicos que se dá na exposição de figuras de Lissajous, que pode ser feita com pêndulos de massa variável. 
Na Física, são exemplos de sistemas oscilatórios: o pêndulo simples, que foi o instrumento usado na fabricação dos primeiros relógios, diapasões, que são objetos usados na afinação de instrumentos musicais, a corrente elétrica, etc.

Problemas que envolvem o estudo de sistemas oscilatórios com massa variável têm sido abordados por outros autores ao longo da última década, por exemplo: (i) um estudo envolvendo o oscilador harmônico com massa variável e a segunda lei de Newton é abordado por Correa et al. (2011), onde tem-se a discussão de um modelo teórico/matemático de um sistema específico envolvendo um garrafa com fluido vazando o qual é confrontado com os resultados experimentais obtidos pelo autores; (ii) já Aguiar e Guedes (2013) se valem do formalismo hamiltoniano para discutir um modelo matemático para osciladores harmônicos amortecidos dependentes do tempo, os chamados osciladores de Lane-Emden e (iii) os autores Nascimento e Guedes (2014) tratam de osciladores clássicos com massa dependente da posição (OMDP) realizando uma correspondência entre eles e o oscilador com massa constante (OMC) através do método de fatoração do hamiltoniano.

Dando continuidade ao tratamento de osciladores com massa variável que também foi trabalhado por outros autores, busca-se, neste texto, uma abordagem do pêndulo com massa variável no tempo com dependência exponencial e polinomial. Enquanto os textos citados acima trabalham com uma abordagem com aparato experimental ou formalismo hamiltoniano, nesta pesquisa, os problemas são modelados e resolvidos por técnicas de resolução de equações diferenciais em que se destaca a modelagem pela equação de Bessel.

\section{Referencial teórico}

A seguir, são apresentados os elementos que compõem o referencial teórico do presente estudo.

\subsection{Equações diferenciais}

Muitos dos problemas são vistos com suas grandezas variando de acordo com o tempo ou outra variável independente, ou seja, esses problemas levam em consideração a taxa com que alguns fatores variam. Dessa maneira, equações diferenciais levam à compreensão e à análise de muitos fenômenos que são modelados por relações que envolvem taxas de variação.

Para Boyce e Diprima (2006), uma equação diferencial pode ser definida como uma expressão matemática que envolve as derivadas de uma ou mais variáveis dependentes em relação a uma ou mais variáveis independentes. Sua solução é uma função que quando substituída, satisfaz a igualdade.

Segundo Çengel e Palm III (2014), equações diferenciais são usadas para investigar uma grande variedade de problemas nas ciências e na engenharia, além de fazerem parte da 
formação educacional dessas áreas. Portanto, compreender as equações diferenciais é um passo para entender o funcionamento de diversos modelos que norteiam as ciências.

Conforme apresentado em Zill (2016), as equações diferenciais são classificadas quanto ao tipo de derivada ao qual essa estão relacionadas, ou seja, quando a equação contiver apenas funções e suas derivadas dependentes de apenas uma variável independente essa é denominada de Equação Diferencial Ordinária (EDO); mas, quando a equação possuir derivadas parciais, ou seja, depender de mais de uma variável independente, ela passa a ser classificada como Equação Diferencial Parcial (EDP).

A ordem de uma equação diferencial é dada pela ordem da derivada de maior ordem presente na equação. Além disso, a equação é linear quando não aparecem produtos ou termos transcendentais da variável dependente ou de suas derivadas. Caso apareçam, a equação é não linear.

Uma equação linear de ordem n é escrita como Boyce e Diprima (2006):

$$
a_{n}(t) \frac{d^{n} y}{d t^{n}}+a_{n-1}(t) \frac{d^{n-1} y}{d t^{n-1}}+\ldots+a_{1}(t) \frac{d y}{d t}+a_{0}(t) y=g(t)
$$

Se $g(t)=0$, a equação é homogênea. Caso contrário, é não homogênea.

Se os coeficientes da Equação (1) são constantes, pode-se escrever:

$$
a_{n} \frac{d^{n} y}{d t^{n}}+a_{n-1} \frac{d^{n-1} y}{d t^{n-1}}+\ldots+a_{1} \frac{d y}{d t}+a_{0} y=g(t)
$$

A equação homogênea associada à Equação (2) é

$$
a_{n} \frac{d^{n} y}{d t^{n}}+a_{n-1} \frac{d^{n-1} y}{d t^{n-1}}+\ldots+a_{1} \frac{d y}{d t}+a_{0} y=0
$$

Para obter a solução da Equação (3), substitui-se a função

$$
y=e^{r t}
$$

em que se obtém:

$$
a_{n} r^{n}+a_{n-1} r^{n-1}+\ldots+a_{1} r+a_{0}=0
$$

A Equação (4) é polinomial e fornece $n$ raízes e tem-se $n$ soluções como a função da Equação (3). A solução da Equação (3) será a combinação linear dessas $n$ soluções

$$
y(t)=c_{1} e^{r_{1} t}+c_{2} e^{r_{2} t}+\ldots+c_{n} e^{r_{n} t} .
$$

A solução para a Equação (2) é a Equação (5) acrescida de uma solução particular a depender de $g(t)$. As constantes $c_{1}, c_{2}, \ldots, c_{n}$ são determinadas a partir de condições iniciais ou de contorno dadas.

Se os coeficientes da equação diferencial linear não são constantes, deve-se recorrer a outros métodos de resolução, dos quais destaca-se o método de Frobenius, que é utilizado quando há pontos singulares regulares na definição das funções que descrevem estes coeficientes.

No que segue, será apresentada uma equação diferencial que utiliza tal método em sua resolução. Devido a inúmeras aplicações e inclusive, será vista neste trabalho, apresenta-se a equação diferencial de Bessel. 
A equação diferencial de Bessel de ordem v pode ser encontrada em Zill (2016) e é definida por

$$
x^{2} \frac{d^{2} y}{d x^{2}}+x \frac{d y}{d x}+\left[x^{2}-v^{2}\right] y=0
$$

Para encontrar a solução da Equação (6), suponha uma série do tipo

$$
y=\sum_{n=0}^{\infty} a_{n} x^{n+r}
$$

Desta forma, estão sendo buscadas soluções em torno de $x=0$, em que $r$ é um valor a ser determinado.

Substituindo a Equação (7) em (6), obtém-se:

$\sum_{n=0}^{\infty} a_{n}(n+r)(n+r-1) x^{n+r}+\sum_{n=0}^{\infty} a_{n}(n+r) x^{n+r}+\sum_{n=0}^{\infty} a_{n} x^{n+r+2}-v^{2} \sum_{n=0}^{\infty} a_{n} x^{n+r}=0$

ou ainda

$$
a_{0}\left(r^{2}-v^{2}\right) x^{r}+x^{r} \sum_{n=0}^{\infty} a_{n}\left[(n+r)^{2}-v^{2}\right] x^{n}+x^{r} \sum_{n=0}^{\infty} a_{n} x^{n+2}=0
$$

Da Equação 9, tem-se a equação indicial $r^{2}-v^{2}=0$, com raízes $r= \pm v$. Quando $r=v$, a Equação (9) fornece $(1+2 v) a_{1}=0$ e a relação de recorrência

$$
a_{2 n}=\frac{(-1)^{n}}{2^{2 n+v} n ! \Gamma(1+v+n)}
$$

em que $a_{0}$ pode ser definido por

$$
a_{0}=\frac{1}{2^{v} \Gamma(1+v)}
$$

e $\Gamma(x)$ é a função Gamma definida por

$$
\Gamma(x)=\int_{0}^{\infty} t^{x-1} e^{-t} d t
$$

Substituindo a Equação (10) na série da Equação (7), tem-se a solução

$$
J_{v}(x)=\sum_{n=0}^{\infty} \frac{(-1)^{n}}{n ! \Gamma(1+v+n)}\left(\frac{x}{2}\right)^{2 n+v}
$$

De modo análogo, para $r=-v$, obtém-se

$$
J_{-v}(x)=\sum_{n=0}^{\infty} \frac{(-1)^{n}}{n ! \Gamma(1-v+n)}\left(\frac{x}{2}\right)^{2 n-v}
$$

Segundo Zill (2016), as funções $J_{v}(x)$ e $J_{-v}(x)$ são as funções de Bessel de primeira espécie e as funções de segunda espécie são dadas por

$$
Y_{v}(x)=\frac{\cos (v \pi) J_{v}-J_{-v}(x)}{\operatorname{sen}(v \pi)}
$$

e a combinação linear

$$
y=c_{1} J_{v}(x)+c_{2} Y_{v}(x)
$$

é solução da Equação (6) para qualquer valor de $v$.

\subsection{Pêndulo simples}

Um pêndulo simples, também chamado de pêndulo matemático, consiste em um modelo idealizado de um oscilador harmônico simples constituído por um corpo puntiforme de massa $m$ suspenso por um fio inextensível e de massa desprezível. Quando o corpo é puxado lateralmente a partir de sua posição de equilíbrio e a seguir liberado, ele oscila em torno dessa posição. 
Algumas situações familiares, como uma bola de demolição presa a um cabo de guindaste ou, uma criança sentada em um balanço, desde que os deslocamentos em relação à posição de equilíbrio sejam pequenos de forma a podermos aproximar o ângulo de seu seno, $\operatorname{sen} \theta \cong \theta$, podem ser tratadas como pêndulo simples.

Considere uma esfera de massa $m$ e raio $R$ presa por um fio de massa desprezível ao um ponto fixo $O$. Seja a distância entre o centro da esfera e o ponto $O$ igual a $l$, com $R \ll l$. Esta hipótese nos permite tratar a esfera como uma massa puntiforme e o objeto físico como um pêndulo simples (para os casos em que $R \cong l$, o objeto seria o que chamamos de pêndulo composto ou pêndulo físico). A seguir, iremos considerar oscilações de pequena amplitude, para um pêndulo simples, e descrever matematicamente o seu movimento.

Figura 1 - Pêndulo simples.

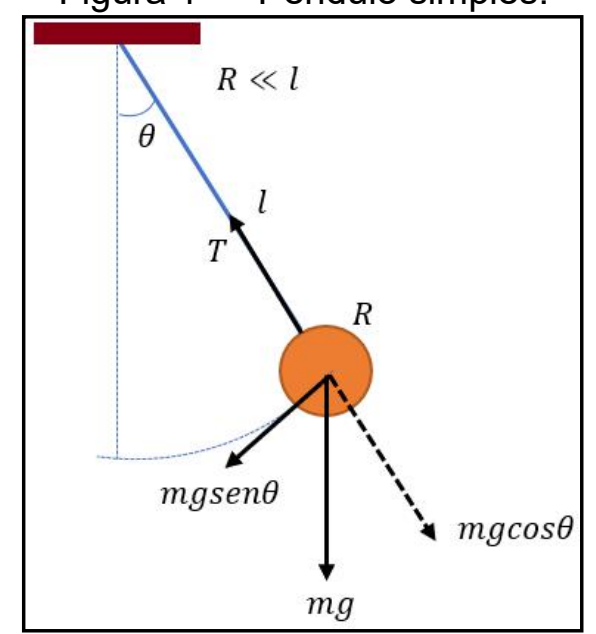

Fonte: Elaboração dos autores (2020).

Aplicando a segunda lei de Newton ao objeto da massa $m$, tem-se

$$
-m g \operatorname{sen} \theta=m \frac{d^{2} s}{d t^{2}} \Rightarrow-g \operatorname{sen} \theta=l \frac{d^{2} \theta}{d t^{2}}
$$

O sinal de menos na equação anterior decorre do fato de o comprimento de arco crescer a partir do equilíbrio, da esquerda para a direita, ao passo que a componente tangencial da força está orientada da direita para a esquerda.

$$
\frac{d^{2} \theta}{d t^{2}}+\frac{g}{l} \operatorname{sen} \theta=0
$$

O desenvolvimento da função seno, envolvida da Equação (18), em série de Taylor nas vizinhanças do ponto 0 (zero), fornece

$$
\operatorname{sen} \theta=\theta-\frac{\theta^{3}}{3 !}+\frac{\theta^{5}}{5 !}-\ldots
$$

Percebe-se que, quando $\theta \leq 10^{\circ}$, pode-se escrever $\operatorname{sen} \theta=\theta$, $\operatorname{com} \theta$ medido em radianos. Para $\theta=10^{\circ}$ comete-se um erro da ordem $0,5 \% \quad\left(\operatorname{sen} 10^{\circ}=0,1736\right)$; o erro aumenta à medida que $\theta$ cresce. Considerando somente pequenos ângulos de oscilação, tem-se $\operatorname{sen} \theta \cong \theta$ e a Equação (18) pode ser reescrita como

$$
\frac{d^{2} \theta}{d t^{2}}+\frac{g}{l} \theta=0
$$


Essa é uma equação diferencial linear de segunda ordem com coeficientes constantes que tem solução

$$
\theta(t)=c_{1} e^{i \sqrt{\frac{g}{l}} t}+c_{2} e^{-i \sqrt{\frac{g}{l} t}}
$$

de forma que as exponenciais podem ser escritas como senos e cossenos e as constantes redefinidas para obter

$$
\theta(t)=\theta_{0} \cos \left(\sqrt{\frac{g}{l}} t+\varphi_{0}\right)
$$

Usando as condições iniciais $\theta(0)=\theta_{\max } \mathrm{e} \frac{d \theta}{d t}(0)=0$, tem-se $\varphi_{0}=0 \mathrm{e}$

$$
\theta(t)=\theta_{\max } \cos (\omega t)
$$

em que foi definido

$$
\omega=\sqrt{\frac{g}{l}}
$$

A Equação (23) é a solução para o pêndulo simples, onde percebe-se que há oscilação de período

$$
T=2 \pi \sqrt{\frac{l}{g}}
$$

e frequência

$$
f=\frac{1}{2 \pi} \sqrt{\frac{g}{l}}
$$

O período $T$ e a frequência $f$ do movimento de um pêndulo simples são independentes da massa do objeto que oscila, uma vez que a massa é constante e não há termos de amortecimento na equação diferencial. Problemas deste tipo descrevem sistemas harmônicos.

\section{Metodologia}

No percurso metodológico para análise e compreensão dos fenômenos descritos neste trabalho, buscou-se uma revisão bibliográfica de equações diferenciais, sua classificação e métodos de resolução. Durante, são vistas as equações como relações entre taxa de variação, bem como os casos de coeficientes constantes e variáveis, em que são apresentados métodos por funções exponenciais e série de potências. Após discutir as equações diferenciais, foi necessário revisar o pêndulo simples que, com massa constante, descreve um movimento oscilatório independente da massa do objeto.

Conhecendo as equações diferenciais e os princípios físicos do pêndulo, sugere-se a análise deste pêndulo com massa variável, considerando as hipóteses de massa com dependência exponencial e em termos de potências da variável tempo.

No caso de variação exponencial, o problema será modelado por uma equação diferencial com coeficientes constantes, em que se deve considerar o caso de redução àquele de massa constante, bem como as soluções dependentes dos parâmetros da função exponencial. 
Quando a variação da massa é do tipo polinomial, após substituição de variáveis, o problema é modelado por uma equação de Bessel, em que a ordem desta equação é dependente do expoente da função massa.

Em todos os casos, são realizadas as interpretações das equações comparadas ao caso de massa constante. De posse das funções que descrevem a posição do objeto, os gráficos foram construídos nos softwares GeoGebra ${ }^{1}$ e $S c i l a b^{2}$, cujas escolhas se basearam no fato de que tais programas são de acesso gratuito e de fácil operacionalização. Os gráficos permitirão uma análise em que são verificadas as amplitudes e fica evidente o amortecimento devido à massa variável.

\section{Resultados}

Considerando o pêndulo com massa variável, tem-se que a segunda lei de Newton fornece:

$$
\frac{d}{d t}\left(m l \frac{d \theta}{d t}\right)=-m g \theta \text {, }
$$

em que o termo em parênteses é o momento linear na direção angular. Esta equação pode ser reescrita como

$$
m \frac{d \theta^{2}}{d t^{2}}+\frac{d m}{d t} \frac{d \theta}{d t}+m \omega^{2} \theta=0
$$

\subsection{Massa com variação exponencial}

Inicialmente, pode-se analisar o caso em que a massa varia exponencialmente em função do tempo, sendo ela dada por $m(t)=m_{0} e^{a t}$.

Quando $a=0$, a massa é constante, no entanto, quando $a>0$, a massa é crescente e quando $a<0$, a massa é decrescente, fato observado por Caccamo e Magazù (2016) ao usar a transformada wavelet para processar o movimento de um objeto pendurado que perde areia fina a uma taxa exponencial. A Figura 2 mostra o gráfico algumas situações.

Figura 2 - Gráfico da massa com variação exponencial.

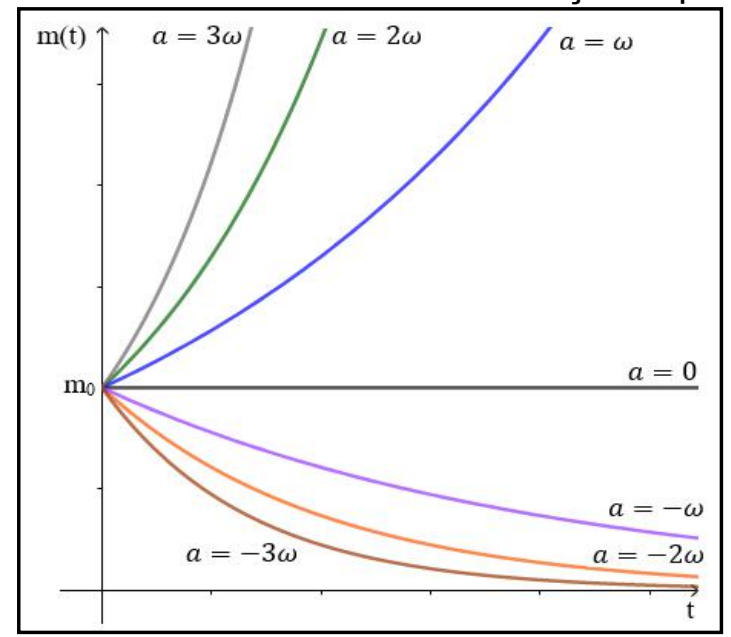

Fonte: Elaboração dos autores (2020).

\footnotetext{
1 https://www.geogebra.org/

2 https://www.scilab.org/
} 
A linha em que $a=0$ indica a massa constante ao longo do tempo, enquanto que as demais curvas indicam a massa variável, em que quando $a$ é positivo, a função é crescente e quando $a$ é negativo, a função é decrescente.

Para este tipo de massa com variação exponencial, a Equação (28), após substituição da massa e sua derivada se torna:

$$
\frac{d^{2} \theta}{d t^{2}}+a \frac{d \theta}{d t}+\omega^{2} \theta=0
$$

Essa é uma equação diferencial linear de segunda ordem com coeficientes constantes, que tem solução:

$$
\theta(t)=c_{1} e^{r_{1} t}+c_{2} e^{r_{2} t}
$$

com $r_{1}=\frac{a}{2}+\frac{1}{2} \sqrt{a^{2}-4 \omega^{2}}$ e $r_{2}=\frac{a}{2}-\frac{1}{2} \sqrt{a^{2}-4 \omega^{2}}$.

Quando $a=0$, com $r_{1}=i \omega$ e $r_{2}=i \omega$ e a solução (30) pode ser reescrita como a Equação (23). Quando $|a|=2 \omega, r_{1}$ e $r_{2}$ são iguais e a solução (30) pode ser reescrita como:

$$
\theta(t)=c_{1} e^{\frac{-a}{2} t}+c_{2} t e^{\frac{-a}{2} t}
$$

Substituindo as mesmas condições de contorno da subseção 2.2 , tem-se:

$$
\theta(t)=\theta_{\text {max }}(1+\omega t) e^{-\omega t}
$$

para $a=2 \omega \mathrm{e}$

$$
\theta(t)=\theta_{\max }(1-\omega t) e^{\omega t}
$$

para $a=-2 \omega$.

Quando $|a|>2 \omega, r_{1}$ e $r_{2}$ são reais e a solução (30) pode ser reescrita como:

$$
\theta(t)=e^{-a t / 2}\left[c_{1} e^{\frac{1}{2} \sqrt{a^{2}-4 \omega^{2}} t}+c_{2} e^{-\frac{1}{2} \sqrt{a^{2}-4 \omega^{2}} t}\right]
$$

Substituindo as condições de contorno da subseção 2.2, tem-se:

$$
\theta(t)=\frac{\theta_{\max }}{2} e^{-a t / 2}\left[\left(1+\frac{a}{\sqrt{a^{2}-4 \omega^{2}}}\right) e^{\frac{1}{2} \sqrt{a^{2}-4 \omega^{2}} t}+\left(1-\frac{a}{\sqrt{a^{2}-4 \omega^{2}}}\right) e^{-\frac{1}{2} \sqrt{a^{2}-4 \omega^{2}} t}\right]
$$

Quando $|a|<2 \omega, r_{1}$ e $r_{2}$ são complexas e a solução (35) pode ser reescrita como:

$$
\theta(t)=e^{-a t / 2}\left[c_{1} \cos \left(\frac{1}{2} \sqrt{4 \omega^{2}-a^{2}} t\right)+c_{2} \operatorname{sen}\left(\frac{1}{2} \sqrt{4 \omega^{2}-a^{2}} t\right)\right]
$$

Substituindo as condições de contorno da subseção 2.2 , tem-se:

$$
\theta(t)=\theta_{\text {max }} e^{-a t / 2}\left[\cos \left(\frac{1}{2} \sqrt{4 \omega^{2}-a^{2}} t\right)+\frac{a}{\sqrt{4 \omega^{2}-a^{2}}} \operatorname{sen}\left(\frac{1}{2} \sqrt{4 \omega^{2}-a^{2}} t\right)\right]
$$

A Figura 3 mostra o gráfico da solução do pêndulo com massa variando exponencialmente com o tempo comparada à solução do pêndulo com massa constante. 
Figura 3 - Gráfico da solução do pêndulo com massa variando exponencialmente.

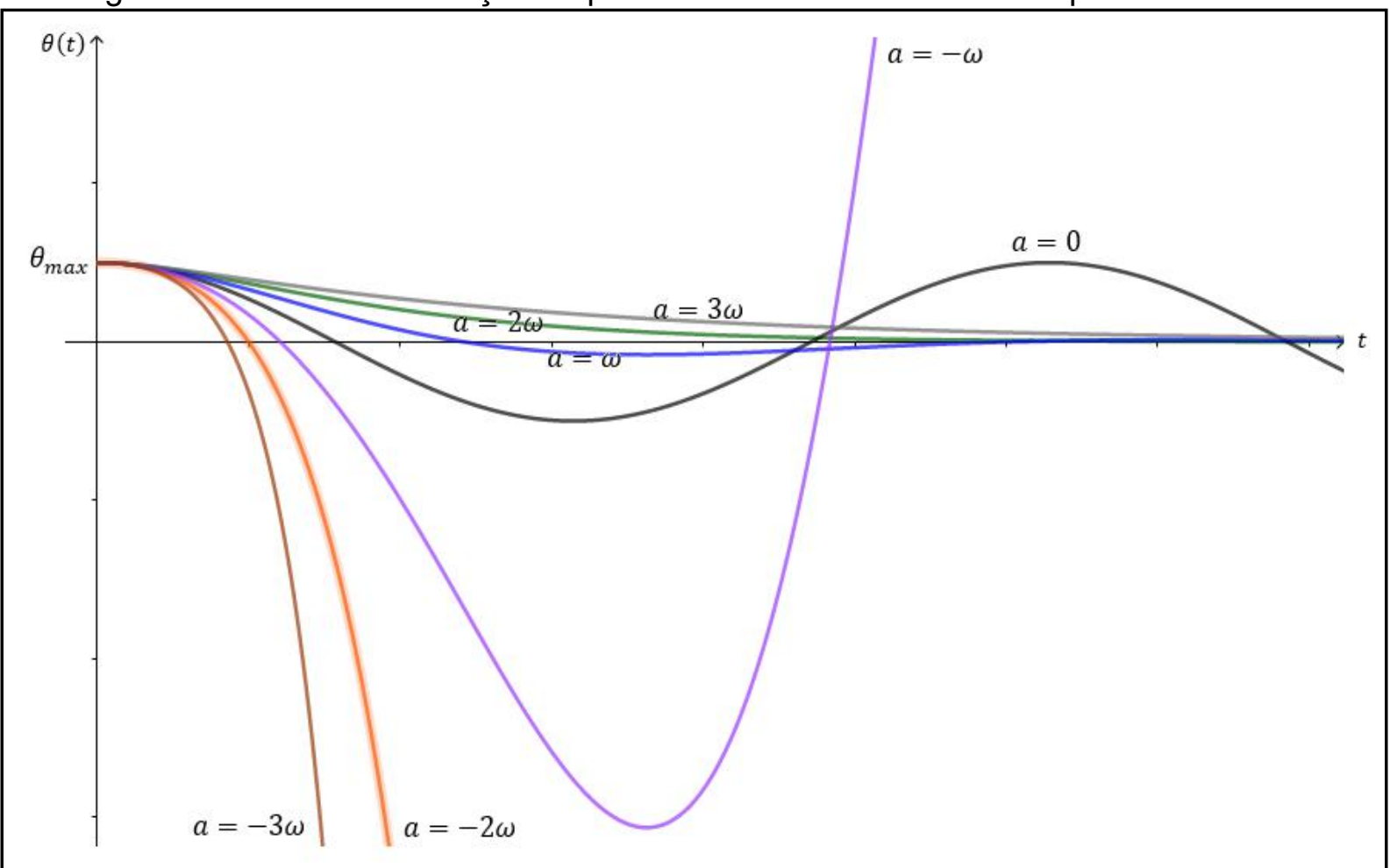

Fonte: Elaboração dos autores (2020).

A Figura 3 mostra o gráfico do ângulo em função do tempo para cada um dos casos analisados, em que os valores de a considerados são os mesmos da Figura 2 quando foi mostrada a variação de massa. A linha de cor preta indica o caso em que a massa é constante e há oscilação. Os casos em que $a$ é positivo (linhas azul, verde e cinza), o ângulo decresce e se aproxima de zero, em que se pode perceber que quanto menor o valor de $a$, mais rapidamente o ângulo decresce. No caso em que $a=-\omega$, o ângulo tente a infinito, mas antes decresce a um valor mínimo. No caso em que $a=-2 \omega$ e $a=-3 \omega$, o ângulo tende a menos infinito.

\subsection{Massa com variação polinomial}

Outro caso a ser analisado é aquele em que a massa depende do tempo da forma $m=$ $m_{0} t^{n}$. Esse caso reflete aquele em que um pêndulo tem massa constante para $n=0$, massa crescente para $n>0$ e massa decrescente para $n<0$. No caso de $n<0$, existe uma singularidade em $t=0$.

A Equação (28) se torna

$$
t \frac{d^{2} \theta}{d t^{2}}+n \frac{d \theta}{d t}+t \omega^{2} \theta=0
$$

Fazendo a substituição

$$
\theta(t)=t^{\frac{1-n}{2}} z(t)
$$

obtém-se

$$
t^{2} \frac{d^{2} z}{d t^{2}}+t \frac{d z}{d t}+\left[t^{2} \omega^{2}-\frac{(n-1)^{2}}{4}\right] z=0
$$


Agora, a substituição

$$
u=\omega t
$$

torna a Equação (40) em:

$$
u^{2} \frac{d^{2} z}{d u^{2}}+u \frac{d z}{d u}+\left[u^{2}-\frac{(n-1)^{2}}{4}\right] z=0
$$

que é uma equação de Bessel de ordem $\frac{n-1}{2}$.

A solução da Equação (42) é dada por

$$
z(u)=c_{1} J_{\frac{n-1}{2}}(u)+c_{2} Y_{\frac{n-1}{2}}(u)
$$

Fazendo a substituição de variável dada pela Equação (41), retorna-se à variável $t$ :

$$
z(t)=c_{1} J_{\frac{n-1}{2}}(\omega t)+c_{2} Y_{\frac{n-1}{2}}(\omega t)
$$

Substituindo a Equação (44) na Equação (39), tem-se a posição angular

$$
\theta(t)=c_{1} t^{\frac{1-n}{2}} J_{\frac{n-1}{2}}(\omega t)+c_{2} t^{\frac{1-n}{2}} Y_{\frac{n-1}{2}}(\omega t)
$$

Para $n=1$, a solução (45) se torna

$$
\theta(t)=\theta_{\max } J_{0}(\omega t)
$$

e para $n=2$, tem-se

$$
\theta(t)=\theta_{\max } \sqrt{\frac{\pi}{2 \omega}} J \frac{1}{2}(\omega t)
$$

que satisfazem as condições iniciais da subseção 2.2. A Figura 4 mostra o gráfico dessas soluções comparadas com a solução de massa constante.

Figura 4 - Gráfico da solução do pêndulo com massa em termos de potências do tempo.

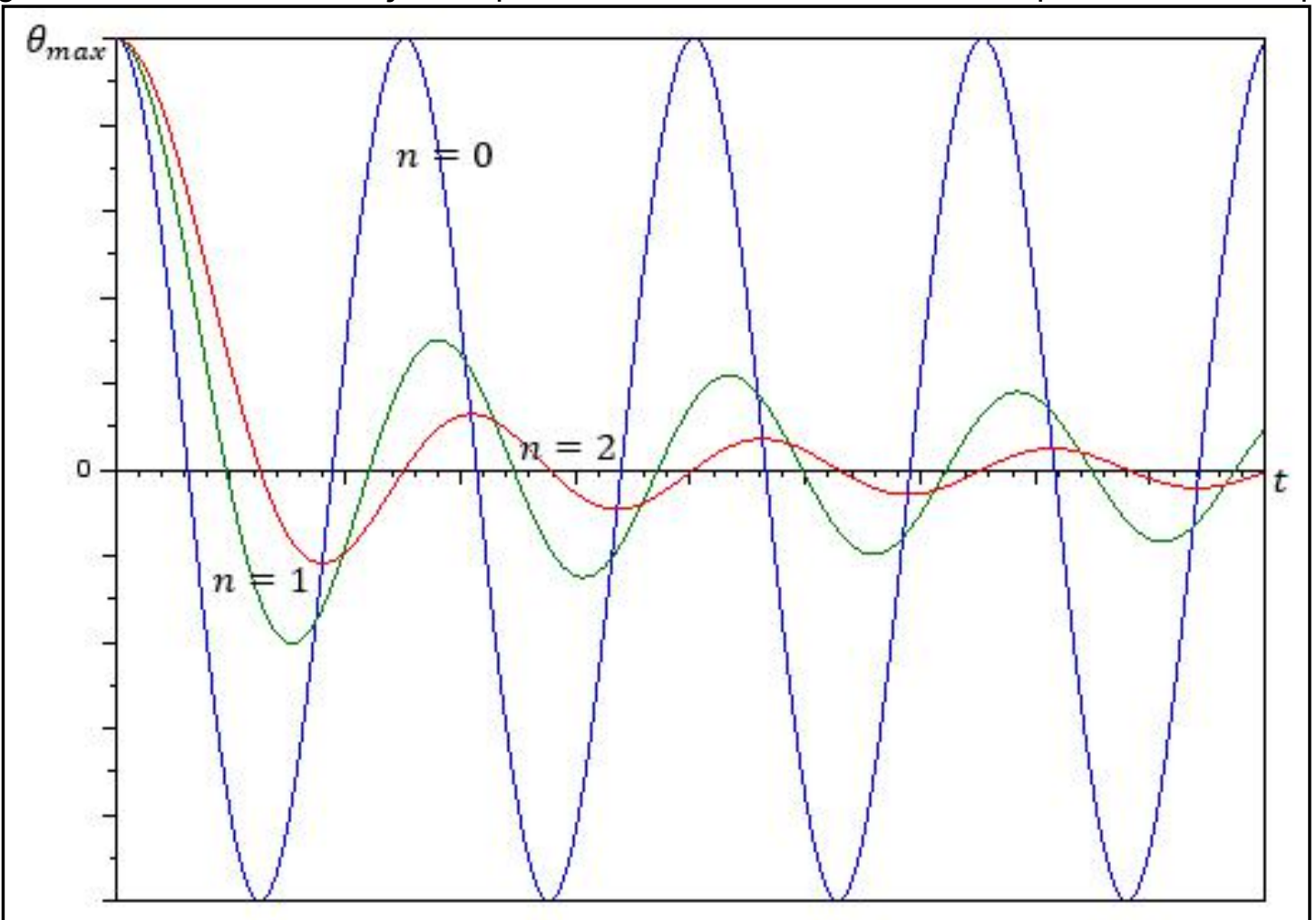

Fonte: Elaboração dos autores (2020). 
O gráfico apresenta as soluções dos três casos considerados. A curva azul é o caso de massa constante e não há amortecimento. Quando a massa é variável, acontece o decaimento e este é cada vez maior, à medida que aumenta o valor de $n$, ou seja, à medida em que a massa aumenta. O decaimento, com redução de amplitude, vem a concordar com Aguiar e Guedes (2013), ao tratar osciladores dependentes do tempo através de formalismo hamiltoniano.

\section{Considerações finais}

Durante o estudo do pêndulo com massa variável, foi utilizada a segunda lei de Newton e as equações diferencias foram empregadas e discutidas na modelagem de um fenômeno físico. Ao considerar a massa com variação exponencial, as soluções da equação mostram que, nos casos em que há perda de massa, não há oscilação, o que também é visto na análise gráfica. Nos casos em que há ganho de massa, a taxa de variação entra no sistema como um fator de decaimento.

Quando a massa foi considerada com dependência em potência do fator tempo, após mudanças de variáveis, a equação do movimento fica modelada pela equação de Bessel que tem soluções em séries de potências. A solução e a análise gráfica mostraram que há um decaimento quanto maior a ordem da equação de Bessel, ou seja, quanto maior o exponente na expressão da massa. Em outros termos, o amortecimento possui relação direta com taxa de variação da massa.

Ao analisar estes casos de massa variável, espera-se que o estudo possa contribuir com a Física Matemática, destacando problemas de não homogeneidade, enriquecendo o campo das aplicações das equações diferenciais.

\section{Referências}

AGUIAR, V.; GUEDES, I. Osciladores harmônicos amortecidos dependentes do tempo. Revista Brasileira de Ensino de Física, São Paulo, v. 35, n. 4, p. 1-9, out./dez. 2013. DOI: http://dx.doi.org/10.1590/S1806-11172013000400011.

BOYCE, William; DIPRIMA, Richard. Equações Diferenciais Elementares e Problemas de Valores de Contorno. 8. ed. Rio de Janeiro: LTC, 2006.

Caccamo, M. T.; Magazù, S. Variable mass pendulum behaviour processed by wavelet analysis. European Journal of Physics. v. 38, n. 1, p. 1-9, 30 nov. 2016. DOI: https://doi.org/10.1088/0143-0807/38/1/015804.

ÇENGEL, Yunus; PALM III, William. Equações Diferenciais. 1. ed. Nova lorque: MCGRAW HILL, 2014.

CORREA, Eberth; ORTIZ, J. S. Espinoza; VALÉRIO, Mauro; DUTRA, Jomhara. Oscilador harmônico com massa variável e a segunda lei de Newton. Revista Brasileira de Ensino de Física, São Paulo, v. 33, n. 4, p. 1-9, out./dez. 2011. DOI: https://doi.org/10.1590/S180611172011000400007.

LENSKI, Richard. Dynamics of interactions between bacteria and virulent bacteriophage. In: Advances in microbial ecology. Boston: Springer, 1988. 
LINDE, Andrei. Inflationary cosmology. Physics Reports, v. 333, p. 575-591, ago. 2000. DOI: https://doi.org/10.1007/978-3-540-74353-8 1.

NASCIMENTO, J. P. G.; GUEDES, I. Osciladores clássicos com massa dependente da posição. Revista Brasileira de Ensino de Física, São Paulo, v. 36, n. 4, p. 1-6, out./dez. 2014. DOI: http://dx.doi.org/10.1590/S1806-11172014000400009.

ROMEO, F. A simple model of energy expenditure in human locomotion. Revista Brasileira de Ensino de Física, São Paulo, v. 31, n. 4, p. 1-5, dez. 2009. DOI: https://doi.org/10.1590/S180611172009000400008.

SALAMANGA, Marcin. An Application of the Harmonic Oscillator Model to Verify Dunning's Theory of the Economic Growth. Statistika: Statistics and Economy Journal, Prague, v. 93, n. 3, p. 5669, set. 2013.

SILVA, Adilson Costa da; HELAYËL NETO, José Abdalla. Simulador de Oscilações Mecânicas. Revista Brasileira de Ensino de Física, São Paulo, v. 38, n. 3, p. 1-14, 7 jun. 2016. DOI: https://doi.org/10.1590/1806-9126-RBEF-2016-0042.

THORNTON, Stephen T.; MARION, Jerry N. Dinâmica Clássica de sistemas e partículas. Tradução da 5a edição norte-americana. São Paulo: Cengage Learning, 2014.

ZILL, Dennis. Equações Diferenciais com aplicações em modelagem. 3. ed. São Paulo: Cengage Learning, 2016. 